\title{
For Sale by Owner: When to Use a Broker and How to Price the House
}

\author{
STEPHEN W. SALANT \\ Department of Economics, University of Michigan, Ann Arbor, MI 48109
}

\begin{abstract}
By using a broker, the owner of a house can speed up his search for buyers but must pay a percentage of the sale price as a commission. Nonstationarities inherent in the housing market may make it optimal to market a house "by-owner" at the outset and to retain a broker only if the house remains on the market later in the selling season. This article investigates the optimal sequence of asking prices within the by-owner phase, within the broker phase, and at the transition between the two phases. The asking price declines within each phase but may jump up at the transition to cover part of the commission. The model implicity determines the demand for broker services as a function of the commission rate. When estimated, it may be useful in investigations of price fixing among brokers.
\end{abstract}

Keywords: Dynamic programming, housing market, real estate broker, search theory

A home owner wishing to sell his house can either utilize the services of a broker or, alternatively, can attempt to sell the house himself. The disadvantage of brokers is well-known: they charge a commission which is typically $6 \%$ of the sales price. There are, however, some offsetting advantages. If the owner has moved to another city, the cost of holding open-houses or showing the house to prospective buyers may be prohibitive. Even if it is not inconvenient for the owner to show the house himself, there remains the problem of calling the availability of the house to the attention of prospective buyers. Without a broker, owners cannot get access to the multiple-listing service. Instead, they must rely on "for sale" signs, classified ads and the neighborhood rumor mill. Since brokers can utilize computerized listings as well as these other channels of communication, they are more likely to contact prospective buyers. Hence, by retaining a broker, the owner can sample prospective buyers more rapidly.

Owners attempting to sell their own houses are often bombarded with fliers, phone calls, and visits from real estate agents eager to get the listing should the owners change their minds. The abundance of these solicitations - as well as direct observation of discouraged sellers - suggests that, after an unsuccessful interval of attempting to sell their own homes, owners frequently do retain brokers.

If a broker is eventually engaged, the question naturally arises as to how the house should be re-priced. On the one hand, the broker may argue that it would be irrational to raise the asking price, since no buyer had been found at the lower price when the owner was selling it himself. This argument is less than compelling, however, since it assumes that every 
prospective buyer was already sampled during the by-owner phase. Even if it is optimal in some circumstances to raise the asking price, nonetheless, there does seem to be a limit on the magnitude of the increase. Intuition suggests that if the seller is at all times rational both about the price he charges and about the time when he engages the broker, then the asking price should never be raised to the point where the seller would earn more from a sale with the realtor than he would have in the by-owner phase. For, if that were ever optimal, bringing in the broker earlier would have permitted the seller not only to sample more frequently but also to get a better price. Finally, there remains the question of whether it is ever optimal to lower the asking price when the broker is retained.

The purpose of this paper is to construct a tractable model which can be used to address these somewhat subtle pricing issues. We consider a nonstationary search model. The nonstationarity arises in the real world because prospective buyers with families typically prefer not to move during the school year. Hence, a seller typically regards himself as having $T$ periods during the late spring and summer to sell his house. If no sale occurs, renting then becomes the preferred option until the following spring or summer when the house is put back on the market... In our analysis, we focus on optimal behavior during a typical selling cycle of given length $T$. Instead of treating the value of renting until the following spring as exogenous and then computing as the induced "bequest" at the end of any selling season the expected value of continuing optimally (the value discounted to that point of renting, then entering the next selling season, possibly renting again, and so on, forever) we simplify by treating the value of continuing beyond the current selling cycle as exogenous. ${ }^{1}$

The assumed finite horizon induces a nonstationarity in the seller's decision problem which causes the expected value of continued search to decline over time. ${ }^{2}$ As we will discover, under some conditions the seller will prefer to sell the house himself when the expected value of continued search is high but will prefer to engage a broker when the expected value of continued search is low. In each period, the seller must decide whether or not to list his house with a broker and what the asking price should be. If the house is ever listed with a broker, we assume it cannot be "unlisted" since standard broker contracts require that the commission be paid for an interval of time (assumed to exceed $T$ ), even if the seller terminates the broker's services. It is assumed that the house is sold to the first sampled buyer willing to pay at least the asking price and that the transaction takes place at the seller's asking price.

We characterize the optimal time to retain a broker and the optimal pricing strategy with and without a broker. Although the asking price monotonically declines over time, both before and after the introduction of the broker, the asking price should be raised above what it would have been in the absence of a broker when the broker is first enlisted. However, this increase covers only part of the commission.

Although a "search model," our model differs in one important respect from the standard model of job search. In the familiar job-search model, the seller (the worker) samples buyers (firms), as does the seller of a house. Once a buyer is encountered, however, the two models differ. In the standard model of the labor market, buyers typically set the price and the seller accepts or rejects it. In the housing market, on the other hand, the seller typically sets the price and it is the buyer who must decide whether to accept it. In the familiar job search model, it is never optimal for a worker to reject a job worth a given 
amount if the expected discounted value of continuing to search optimally is strictly smaller. In our model, the seller sets the asking price with incomplete information about what the current buyer will accept or reject. It is then always optimal to set the asking price higher than the expected discounted value of continued search.

In the past, antitrust authorities have expressed concern that the seemingly unvarying $6 \%$ commission rate reflects collusion at the national or at least the local level (Federal Trade Commission, 1984). ${ }^{3}$ These hypotheses could in principle be tested, given a derived demand curve for broker services. Properly refined and estimated, our model can provide such derived demands. Hence, it may eventually be helpful in addressing this important question in industrial organization.

\section{The value of optimal behavior}

We consider the following model. In each period, the seller can engage a broker, if he has not done so already. If a broker has been engaged previously, we assume he must be retained. Given the presence or absence of a broker, the seller then sets an asking price for the period and awaits a buyer. We assume that the length of each period is sufficiently short that, at most, one buyer will arrive. Moreover, the probability that a buyer arrives is assumed to be strictly higher if a broker has been engaged. Given that a buyer arrives, however, the likelihood of his having a reservation price in any interval is assumed to be the same with or without a broker. That is, a broker is viewed as someone who speeds up the sampling process-not as someone who brings in better prospects. If a buyer arrives and if he is willing to pay more than the seller asks, then a sale takes place at the seller's asking price. If, however, no buyer arrives or, alternatively, if one does arrive but is unwilling to pay the asking price, the house remains unsold and the next period is entered. At that time, the seller who has so far tried to sell his house himself can once again exercise his option to engage a broker and so on ... If the house has not been sold after $T$ periods, the seller accepts an exogenous terminal bequest-for example, the discounted value of renting. It is assumed that the seller is risk neutral, discounts future payoffs at rate $\beta$ and pays a fixed percentage of the price as a commission if a sale occurs with a broker engaged. ${ }^{4}$

We adopt the following notation:

$V_{t}$ is the maximized expected value to the seller if he enters period $t$ and uses a broker;

$P_{t}$ is the asking price in period $t$ with a broker engaged;

$\alpha$ is the fraction $(\alpha \in(0,1))$ of the price retained by the seller if the house is sold by the broker ( $\alpha$ is typically $94 \%$ );

$\gamma_{S}$ is the probability per period that one buyer is sampled when no broker is engaged;

$\gamma_{R}$ is the probability per period that one buyer is sampled when a broker is engaged;

$g(y)$ is the probability density of reservation prices with or without a broker; $h(p)$ is the hazard function associated with this density of reservation prices, $\left(g(p) / \int_{p}{ }^{u} g(y) d y\right)$;

$[l, \mathrm{u}]$ is the support of the density;

$W_{t}$ is the expected value to the seller if he enters period $t$ with no broker engaged previously (through $t-1$ ) and proceeds optimally;

$\hat{P}_{t}$ is the asking price in period $t$ if, in period $t$, the owner does not utilize a broker; 
$\bar{V}$ is the terminal bequest if there is no sale up through period $T$.

We begin by analyzing the optimal policy when a broker is engaged. In that case,

$V_{t}=\max _{u \geq P_{t} \geq l}\left\{\alpha P_{i} \gamma_{R} \int_{P_{t}}^{u} g(y) d y+\beta V_{t+1}\left[1-\gamma_{R} \int_{P_{t}}^{u} g(y) d y\right]\right\} t=1, \ldots, T$

and

$$
V_{T+1}=\bar{V}
$$

That is, the expected value at $t$ when a broker is engaged equals the probability-weighted sum of the payoff $\left(\alpha P_{t}\right)$ from selling the house today and the payoff $\left(\beta V_{t+1}\right)$ from continuing. To sell the house at $t$, the seller must (a) sample a buyer and (b) draw someone willing to pay at least the asking price. Defining $\gamma_{R} g(y)=f(y)$, we can simplify:

$V_{t}=\max _{\mathrm{u} \geq P_{t} \geq l}\left\{\alpha P_{t} \int_{P_{t}}^{u} f(y) d y+\beta V_{t+1}\left[1-\int_{P_{t}}^{u} f(y) d y\right]\right\} \quad t=1, \ldots, T$

and

$$
V_{T+1}=\vec{V}
$$

Define the function $R(V)$ as follows:

$R(V)=\max _{\mathrm{u} \geq P \geq l}\left\{\alpha P \int_{P}^{u} f(y) d y+\beta V\left[1-\int_{P}^{u} f(y) d y\right]\right\}$

$R\left({ }^{\circ}\right)$ exists and is single-valued since $P$ lies in a compact set and the objective function (in braces) is continuous. Moreover, by the "theorem of the maximum," $R(V)$ is continuous. Denote by $P^{*}$ a maximizer of (2). To emphasize that $P^{*}$ depends implicitly on $V$ we shall sometimes write $P^{*}(V)$. We can rewrite (1) compactly as follows:

$$
\begin{aligned}
V_{t}= & R\left(V_{t+1}\right) \quad t=1, \ldots, T \\
V_{T+1} & =\bar{V}
\end{aligned}
$$

The sequence $\left\{V_{t}\right\}$ is, therefore, generated by a first-order, nonlinear difference equation. To analyze this equation, we note some properties of $R(V)$. Clearly, $R(0)>0$ since even a nonoptimal asking price, $P \in(l, u)$, would result in a strictly positive expected payoff. Moreover, $R(\alpha u)<\alpha u$ since $R(\alpha u)$ would be the weighted average of two terms, each of 
which is strictly smaller than $\alpha u$. Finally, when the derivative exists, $R^{\prime}(V) \in(0, \beta]$ since, in principle, the maximizer $P^{*}(V)$ must be either interior or at the upper or lower limit of the control set. When the derivative exists,

$$
R^{\prime}(V)= \begin{cases}\beta\left[1-\int_{P \cdot(V)}^{u} f(y) d y\right] & \text { if } P^{*}(V) \in(l, u) \\ 0 & \text { if } P^{*}(V)=l \\ \beta & \text { if } P^{*}(V)=u\end{cases}
$$

As we will show later, only the first case is relevant since $P *(V) \in(l, u)$.

$R(\cdot)$ is depicted in Figure 1. As we have seen, the curve is single-valued, continuous, and non-decreasing with a positive vertical intercept and a slope strictly less than $1 .^{5}$

These properties imply that $R(V)$ has a unique fixed point, $V^{*}=R\left(V^{*}\right)$. Moreover, since $R(0)>0$ but $R(\alpha u)<\alpha u, V^{*} \in(0, \alpha u)$. Assuming that $0<\bar{V}<V^{*}$, the induced sequence of $V$ 's $\left(\bar{V}, R(\bar{V}), R^{2}(\bar{V}) \ldots\right)$ strictly increases as we work backwards from $\bar{V}^{6}$ Hence, the value of continuing optimally would decline as time runs forward:

$$
V_{t+1}<V_{t} .
$$

We now investigate the associated sequence of asking prices, $\left\{P_{t}\right\}$ where $P_{t}=P^{*}\left(V_{t+1}\right)$. Differentiating the right-hand side of (2) with respect to $P$ (using Leibniz's rule), we obtain the following first-order condition which must hold if $P *$ is optimal:

a. $P^{*} \in(l, u)$ and $\alpha \int_{P}^{u} f(y) d y-\alpha P f(P)+\beta V f(P)=0$

or b. $P^{*}=l$ and $\alpha+\beta V f(l) \leq 0$

or c. $P^{*}=u$ and $\beta V-\alpha u \geq 0$.

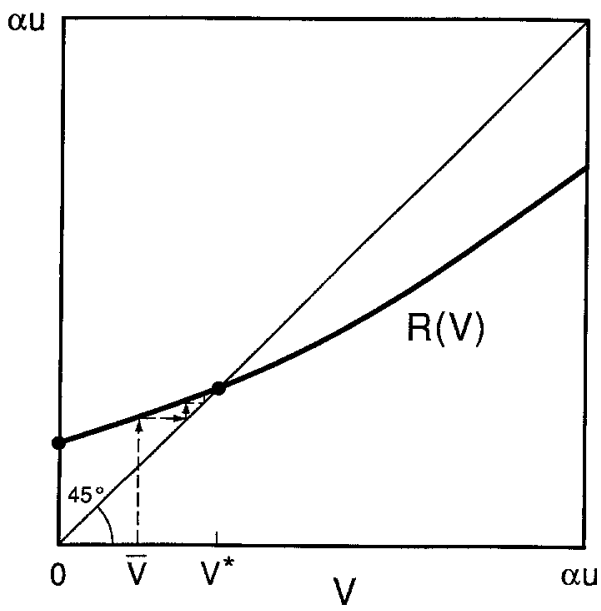

Figure 1. How the value of continuing changes during broker phase. 
Since in our application $V \in(0, \alpha u)$, neither (b) nor (c) can hold. Hence, the optimal asking price is always an interior solution and must satisfy (a). Recall that $h(p)=g(p) / \int_{p}^{u} g(y) d y$. That is, $h(p)$ is the hazard function of the reservation price density. Since $\gamma_{R} g(y)=f(y)$, $h(p)=f(p) / \int_{p}^{u} f(y) d y$. We can therefore rewrite (a) as follows:

$$
f(P)\left\{\frac{\alpha}{h(P)}-\alpha P+\beta V\right\}=0 .
$$

If $h^{\prime}(P)>-h^{2}(P)$, the maximand is strictly concave and this first-order condition has a unique solution, $P^{*}(V)$.

Hence, if the density has a hazard function which increases (or does not decrease too rapidly), then the second-order condition is satisfied for all $P$. Many common densities - among them the uniform, exponential and normal - satisfy this second-order condition for all $P$. We will assume henceforth that it is satisfied globally.

Given the sequence $\left\{V_{t}\right\}$, we can now investigate optimal decision-making when no broker has so far been enlisted. In that case,

$$
\begin{aligned}
W_{t}= & \max \left[V_{t}, \max _{u \geq \hat{P}_{t} \geq l} \hat{P}_{t} \gamma_{S} \int_{\hat{P}_{t}}^{u} g(y) d y+\beta W_{t+1}\left[1-\gamma_{S} \int_{\hat{P}_{t}}^{u} g(y) d y\right]\right] t=1, \ldots, T \\
& \text { and } W_{T+1}=\bar{V} .
\end{aligned}
$$

If the seller enlists a broker at $t$, he receives $V_{t}$. If he does not enlist a broker at $t$, he receives the probability-weighted average of the payoff if he sells his house this period (without a broker) and the payoff expected if $t+1$ is entered without a broker. To sell his house this period, he must (a) sample a buyer and (b) draw someone willing to pay at least the asking price. Define $\gamma=\gamma_{S} / \gamma_{R}$. By assumption, $\gamma \in(0,1)$. We can rewrite the foregoing equation as:

$W_{t}=\max \left[V_{t}, \max _{u \geq \hat{P}_{t} \geq l} \hat{P}_{t} \gamma \int_{\hat{P}_{t}}^{u} f(y) d y+\beta W_{t+1}\left[1-\gamma \int_{\hat{P}_{t}}^{u} f(y) d y\right]\right] t=1, \ldots, T$

Using (8) and the sequence $\left\{V_{t}\right\}$, we can work backwards from $\bar{V}$ to construct the sequence $\left\{W_{t}\right\}$. Three possibilities may arise: (1) $W_{1}=V_{1}$, (2) $W_{t}>V_{t}$ for all $t$, or (3) $W_{1}>V_{1}$ but $W_{t}=V_{t}$ for some $t$. In the first case, it is optimal to use a broker from the outset. In the second case, it is optimal to sell "by owner" throughout the season. In the third case, it is optimal to sell by owner at the outset but to switch to a broker at a certain date if the house remains unsold at that point. Suppose, given the constructed sequences, that case (3) arose. Let $t^{*}$ denote the earliest date at which $W_{t}=V_{t}$. Then the broker should be retained at $t^{*}$. By definition of $t^{*}$,

$$
\begin{aligned}
& W_{t}=V_{t} \text { if } t=t^{*} \\
& W_{t}>V_{t} \text { if } t=1, \ldots, t^{*}-1 .
\end{aligned}
$$


Define the function $S(W)$ as follows:

$$
S(W)=\max _{u \geq \hat{P} \geq l}\left\{\gamma \hat{P} \int_{\hat{P}}^{u} f(y) d y+\beta W\left[1-\gamma \int_{\hat{P}}^{u} f(y) d y\right]\right\} .
$$

Denote by $\hat{P}^{*}(W)$ the maximizer. We can rewrite (8) compactly as:

$$
W_{t}=\max \left(V_{t}, S\left(W_{t+1}\right)\right)=\max \left(R\left(V_{t+1}\right), S\left(W_{t+1}\right)\right) \text { and } W_{T+1}=\bar{V} .
$$

A condition sufficient for the owner to avoid engaging a broker at any time is that $\alpha \leq$ $\gamma$. For, comparing (10) and (2), it can be shown that $S(W)>R(W)$.

To verify this, let $P^{*}(W)$ denote the optimal asking price if the value of continuing is $W$ and a broker is already engaged. Then, from (2),

$$
R(W)=\alpha\left[P^{*}(W) \int_{P^{*}(W)}^{u} f(y) d y\right]+[\beta W]\left\{1-\int_{P^{*}(W)}^{u} f(y) d y\right\} .
$$

Since this asking price might not be optimal if no broker is yet engaged (with the value of continuing again $W$, (10) implies:

$$
S(W) \geq \gamma\left[P^{*}(W) \int_{P^{*}(W)}^{u} f(y) d y\right]+[\beta W]\left\{1-\gamma \int_{P^{*}(W)}^{u} f(y) d y\right\}
$$

Compare the right-hand sides of these two equations. Note that the two factors in square brackets in the upper equation are identical to the two factors in square brackets in the lower equation. When $\gamma \geq \alpha$, the first term of the lower equation must be at least as large as the first term of the upper equation. Moreover, since $\gamma<1$, the second term of the lower equation must strictly exceed the second term of the upper equation (provided $W>0$ and $P^{*}(W)<u$ ). It follows that $S(W)>R(W)$. Thus, the seller would not retain a broker in the last period if he had not engaged one earlier. Moreover, in the penultimate period, the owner would not engage a broker. For, even if he were forced to engage one in the last period, selling by owner in the penultimate period would be more profitable than engaging a broker in the penultimate period. Since, in fact, the seller is free to sell in the final period without a broker and would choose to do so, the advantage to a by-owner sale in the penultimate period is even greater. Repeating this argument at every stage, it follows that the seller would never want to engage a broker if $\alpha \leq \gamma$.

Suppose, on the other hand, that $S(\bar{V})<R(\bar{V})$ (for which a necessary condition is that $\alpha>\gamma)$. Then, even if no broker had been engaged prior to the final period, it would be optimal to retain one in the last period. Hence, the condition is sufficient for a broker to be enaged sometime. If, in addition, $S\left(V^{*}\right)>R\left(V^{*}\right)=V^{*}$ and the time horizon is sufficiently long, by-owner sales will occur at the outset and the broker will be engaged only subsequently. For, if not, the value of entering the initial period with a broker engaged would be approximately $V^{*}$ (the fixed point of $R(\cdot)$ ) while the value of entering without one would be strictly higher. 
Recall that once a broker is enlisted, the value of continuing strictly declines (equation 5 ) as time runs forward. A similar phenomenon occurs during the by-owner phase. To see this, recall that $W_{t^{*}}=V_{t^{*}}$. Since $W_{t^{*}-1}>V_{t^{*}-1}$, equation (11) implies that $S\left(V_{t^{*}}\right)>R\left(V_{t^{*}}\right)$. But $R\left(V_{t^{*}}\right)>V_{t^{*}}$. Therefore,

$$
S\left(V_{t^{*}}\right)>V_{t^{*}}
$$

Now $S(\cdot)$ is monotone: if $c>b, S(c)>S(b) .{ }^{7}$ Hence, by applying $S(\cdot)$ iteratively to the left and right sides of (12), we obtain:

$$
S^{k+1}\left(V_{t^{*}}\right)>S^{k}\left(V_{t^{*}}\right)
$$

But from (9) and (11)

$$
S^{k}\left(V_{t^{*}}\right)=W_{t^{*}-k} \quad \text { for } k=1, \ldots, t^{*}-1 \text {. }
$$

Hence, we have established that $W_{t^{*}-k-1}>W_{t^{*-k}}$ for $k=1, \ldots, t^{*}-1$. As in the second phase, the value of continuing strictly decreases prior to $t^{*}$.

Differentiating the right-hand side of (10) with respect to $\hat{P}$ (using Leibniz's rule), we obtain the following first-order condition which must hold if $\hat{P}^{*}$ is optimal:

$$
\begin{array}{cl}
\text { a. } \hat{P}^{*} \in(l, u) \text { and } \int_{\hat{P}}^{u} f(y) d y-\hat{P} f(\hat{P})+\beta W f(\hat{P})=0 \\
\text { or b. } \hat{P}^{*}=l \quad \text { and } 1+\beta W f(l) \leq 0 \\
\text { c. } \hat{P}^{*}=u \quad \text { and } \beta W-u \geq 0 .
\end{array}
$$

It is straightforward to show that $W_{t} \in(0, u)$. For, equation (12) implies that $S(0)>0$ and $u>S(u)$. Moreover, $0<V_{t^{*}}<u$. Since $S(\cdot)$ is monotone, it follows that $S^{k}\left(V_{t^{*}}\right)$ $\epsilon(0, u)$. Since $W_{t} \in(0, u)$, neither (b) nor (c) can hold. The asking price $\left(\hat{P}^{*}\right)$ is always an interior solution and must satisfy (a). We can rewrite (a) as follows:

$$
f(\hat{P})\left\{\frac{1}{h(\hat{P})}-\hat{P}+\beta W\right\}=0 .
$$

As before, if $h^{\prime}(P)>-h^{2}(P)$, the maximand is strictly concave and (a) has a unique solution $(\hat{P} *(W)$ is a function rather than a correspondence).

In the next section we investigate properties of the optimal sequence of asking prices in the by-owner phase, the broker phase, and at the transition. We then illustrate our findings by means of an example.

\section{Properties of optimal asking prices}

In both the by-owner phase and the broker phase, the optimal asking price always exceeds the discounted expected value from continuing. In the by-owner phase, $P_{t}^{*}>\beta W_{t+1}$; and in the broker phase, $\alpha \hat{P}_{t}^{*}>\beta V_{t+1}$. These conclusions follow directly from the pair of 
first-order conditions (equations (7) and (14)). The intuition behind this result may not be immediately obvious; it implies, for example, that if the owner randomly sampled a buyer with reservation price $y^{*} \in\left(\beta W_{t+1}, \hat{P}_{t}^{*}\right)$, no sale would occur, despite the fact that the seller expects a lower payoff from continuing optimally.

To understand this result, suppose the seller in the by-owner phase did ask only $\beta W_{t+1}$ in period $t$. Then his payoff would be the same whether or not he sold the house at $t$, and variations in the complementary probability weights would be of no consequence. Now suppose he raised his asking price marginally. Although the induced change in the probability weights would not alter the expected payoff, the expected payoff would nonetheless increase because any sales which transpired would occur at a higher price. Hence, it is optimal to precommit to an asking price at $t$ that is strictly higher than the discounted value expected at $t+1$. Like any price-setter constrained to set a uniform price (e.g. a textbook monopolist), our house seller willingly abandons some potential sales which would be profitable so as to enhance the profitability of other potential sales.

This tradeoff is forced on him because he lacks the information necessary to price discriminate. In particular, he does not observe each buyer's willingness to pay. If he did, the seller would in each period demand the larger of two amounts: the expected discounted value of continuing and the current buyer's reservation price. Therefore, the seller would never let a buyer escape whose reservation price exceeded his own expected discounted value of continuing and would fully extract the surplus of the purchaser.

It is interesting to note that no corresponding results appear in the standard model of job search with which most readers are familiar because of the timing assumed in that model. In the standard job-search model, the seller (the worker) moves after the buyer (the firm) and either accepts or rejects the buyer's observed bid. In these circumstances, it is optimal for the seller to accept any bid which exceeds the expected discounted value of continuing. Hence, the unemployed seller of labor services never rejects a bid if his expected discounted value of continuing is lower - much like the price discriminator. But since the bid of the buyer is smaller than his reservation price, the seller does not extract all of the buyer's surplus.

In the last section, we verified that the value of continuing optimally strictly decreases during each phase as time runs forward. Differentiating the conditions which define the optimal asking price in each phase (respectively, equation (6a) and (13a)), we obtain:

$$
\begin{gathered}
P^{* \prime}(V) \frac{\beta / \alpha}{\Delta\left(P^{*}\right)}>0 \\
\hat{P}^{* \prime}(W)=\frac{\beta}{\Delta\left(\hat{P}^{*}\right)}>0,
\end{gathered}
$$

where $\Delta(x)=1+h^{\prime}(x) / h^{2}(x)>0$. Since during the by-owner phase $P_{t}^{*}=P^{*}\left(V_{t+1}\right)$ while in the broker phase $\hat{P}_{t}^{*}=\hat{P}^{*}\left(W_{t+1}\right)$, the asking price within each phase must strictly decrease as time runs forward.

Of particular interest is the behavior of the optimal asking price at the transition between the two phases. We consider the following question: Is the final price in the by-owner 
phase $\left(\hat{P}_{t^{*-1}}^{*}\right)$ higher or lower than the price one period later $\left(\boldsymbol{P}_{t^{*}}^{*}\right)$ when the broker is retained?

It is convenient to think about this question in two steps. In the first step, we compare the final price in the by-owner phase to the price which would then (at $\left.t^{*}-1\right)$ have been optimal if the broker had been retained. That is, we consider how the asking price would have changed if the broker had been engaged without time elapsing. We then compare this hypothetical price, the optimal asking price at time $t^{*}-1$ with the broker engaged, to the optimal price one period later.

As to the first step, it is straightforward to show that:

$$
\alpha P_{t^{*}-1}^{*}<\hat{P}_{t^{*}-1}^{*}<P_{t^{*}-1}^{*} .
$$

That is, if a broker were enlisted at $t^{*}-1$ (one period before it is optimal), the seller should raise the asking price above what he would otherwise charge; but this markup in the asking price would be insufficient to cover the broker's commission.

To verify this claim, recall that $W_{t^{*}}=V_{t^{*}}$. At $t^{*}-1$, therefore, the seller will ask $\hat{P}_{t^{*}-1}$, solving equation (14):

$$
\frac{1}{h\left(\hat{P}_{t^{*}-1}\right)}-\hat{P}_{t^{*}-1}+\beta V_{t^{*}}=0 .
$$

If, instead, he were to utilize a broker at $t^{*}-1$, it would be optimal to ask $P_{t^{*-1}}$, solving equation (7):

$$
\frac{1}{h\left(P_{t^{*}-1}\right)}-P_{t^{*}-1}+\frac{\beta V_{t^{*}}}{\alpha}=0
$$

A comparison of (15) and (16) indicates that $P_{t^{*-1}}>\hat{P}_{t^{*-1}}$. Therefore, if the broker were enlisted at $t^{*}-1$, the asking price would be higher than is optimal with no broker.

Indeed, (15) and (16) imply that:

$$
\hat{P}_{t^{*}-1}-\alpha P_{t^{*}-1}=\frac{1}{h\left(\hat{P}_{t^{*}-1}\right)}-\frac{\alpha}{h\left(P_{t^{*}-1}\right)} .
$$

Hence, if $h(\cdot)$ is increasing, $\hat{P}_{t^{*}-1}>\alpha P_{t^{*}-1}$.

We now proceed to the second step. The optimal asking price at $t^{*}-1$ strictly exceeds the asking price one period later since, once the broker is engaged, the asking price monotonically decreases. The price therefore jumps up in the first step and declines in the second step. This suggests what we now verify: that the asking price can either rise or fall between the final period of the by-owner phase and the first period of the broker phase.

Since $V_{t^{*}}=R\left(V_{t^{*}+1}\right)$, we can rewrite equation (15) as:

$$
\frac{1}{h\left(\hat{P}_{t^{*}-1}\right)}-\hat{P}_{t^{*}-1}+\beta R\left(V_{t^{*}+1}\right)=0 .
$$


Moreover, the price in the first period of the broker phase must satisfy:

$$
\frac{1}{h\left(P_{t^{*}}\right)}-P_{t^{*}}+\frac{\beta V_{t^{*}+1}}{\alpha}=0 .
$$

Hence, assuming that a broker is retained at $t^{*}$, the asking price then will strictly exceed the price one period earlier if and only if $V_{t^{*}+1} / \alpha>R\left(V_{t^{*}+1}\right)$; it will decline if the inequality is reversed.

Either case can be constructed for any $f(y), \beta$, and $\alpha$. To produce an upward jump, one merely sets $\bar{V}$ within $\alpha$ percent of $V^{*}$ and adjusts $\gamma$ so that it is optimal to switch to a broker after a by-owner phase. ${ }^{8}$

To produce a decline in price for any $f(y), \beta$, and $\alpha$, one must set $\bar{V}$ such that $R(\bar{V})$ lies below $\alpha$ percent of $V^{*}$. One can then set $\gamma$ so that the transition occurs to a broker while $V$ remains below this boundary.

To summarize, when the broker is first retained, the asking price can be either higher or lower than it was in the previous period. The change in the asking price at the transition point is the net effect of two opposing tendencies, each of which we have studied in isolation. If the broker were enlisted prematurely at $t^{*}-1$, the asking price would necessarily jump up then. But the asking price in the broker phase decreases between period $t^{*}-$ 1 and period $t^{*}$.

Parameter values chosen for plausibility produce an upward jump in the asking price when the broker is first retained. Consider the benchmark case reported in Table 1. As indicated at the bottom of the table, the broker's commission is assumed to be $6 \%$; the discount factor (per week) is 1; buyer reservation prices are assumed to be uniformly distributed between 150 and 220 (thousand dollars); the probability of drawing a buyer in a given week with no broker is assumed to be $25 \%{ }^{9}$, and the terminal bequest is 183.6 (thousand dollars). The seller is assumed to have at most 17 weeks to sell his house ( $T$ $=17$ ). If a house still remains unsold at that point, the seller collects the terminal bequest.

The optimal strategy is to begin selling by-owner at an asking price of $\$ 207,970$ (which exceeds the discounted value of continuing, $\$ 196,150$ ). The seller should then drop the price each week if no one purchases it. After five weeks, the owner should charge \$207,260. On the following week (week 6), he should retain a broker and initially ask \$213,240 an upward jump of $2.9 \%$. The price jump is insufficient to cover the entire commission. In this example, the final asking price in the broker phase is $\$ 207,560$ - not significantly lower than the owner charged at the outset. Table 2 illustrates the case where the price declines when the broker is introduced. As before, the parameters are listed at the bottom of the table. Note the low value for $\bar{V}$. In this example, the broker is retained only for the final two weeks of the season. The price drops from $\$ 189,990$ on the final week of the by-owner phase to $\$ 186,050$ in the initial week of the broker phase - a decline of $2.1 \%$. We can regard this $\$ 3,940$ decline as the result of two changes. If the broker had been introduced a week earlier, the price would have then been $\$ 4,210$ higher $(\$ 194,200)$. However, in the following period of the broker phase, the price would have declined by $\$ 8,150$ (to $\$ 186,050$ ) so that, on balance, the price declines by $\$ 3,940$. We illustrate each case in the two panels of Figure 2: 
Table 1. Base case simulation with $6 \%$ commission. Upward jump in price when broker is retained.

\begin{tabular}{ccccccc}
\hline & & & & Broker & Self & \\
Periods & $\mathrm{W}$ & $\mathrm{R}$ & $\mathrm{S}$ & $\mathrm{P}$ & $\hat{\mathrm{P}}$ & Phase* \\
\hline 1 & 196.47 & 196.29 & 196.47 & 214.17 & 207.97 & 1.00 \\
2 & 196.15 & 196.03 & 196.15 & 214.01 & 207.81 & 1.00 \\
3 & 195.81 & 195.74 & 195.81 & 213.85 & 207.63 & 1.00 \\
4 & 195.46 & 195.43 & 195.46 & 213.67 & 207.45 & 1.00 \\
5 & 195.09 & 195.09 & 195.09 & 213.46 & 207.26 & 1.00 \\
6 & 194.71 & 194.71 & 194.69 & 213.24 & 207.05 & 0.00 \\
7 & 194.29 & 194.29 & 194.25 & 212.99 & 206.81 & 0.00 \\
8 & 193.82 & 193.82 & 193.76 & 212.72 & 206.55 & 0.00 \\
9 & 193.30 & 193.30 & 193.20 & 212.41 & 206.26 & 0.00 \\
10 & 192.72 & 192.72 & 192.58 & 212.06 & 205.94 & 0.00 \\
11 & 192.07 & 192.07 & 191.88 & 211.67 & 205.57 & 0.00 \\
12 & 191.33 & 191.33 & 191.08 & 211.22 & 205.15 & 0.00 \\
13 & 190.48 & 190.48 & 190.16 & 210.70 & 204.66 & 0.00 \\
14 & 189.51 & 189.51 & 189.10 & 210.10 & 204.10 & 0.00 \\
15 & 188.38 & 188.38 & 187.86 & 209.40 & 203.44 & 0.00 \\
16 & 187.06 & 187.06 & 186.38 & 208.57 & 202.65 & 0.00 \\
17 & 185.49 & 185.49 & 184.61 & 207.56 & 201.71 & 0.00 \\
& 183.60 & 183.60 & 183.60 & & & \\
\hline
\end{tabular}

*Periods when it is optimal to enlist a broker are denoted by " 0.00 "; periods when it is optimal to continue with no broker are denoted by " 1.00 ". Parameters: $\alpha=0.94 ; \beta=1.00 ; \mathrm{u}=220.00 ; 1=150.00 ; \gamma=0.25$; $\overline{\mathrm{v}}=183.60$.

Table 2. Exceptional case-because of low bequest no upward jump in price when broker is retained.

\begin{tabular}{ccccccc}
\hline & & & & Broker & Self & \\
Periods & W & $\mathrm{R}$ & $\mathrm{S}$ & $\mathrm{P}$ & $\hat{\mathbf{P}}$ & Phase* \\
\hline 1 & 202.27 & 195.73 & 202.27 & 212.63 & 210.67 & 1.00 \\
2 & 201.53 & 195.19 & 201.53 & 212.31 & 210.26 & 1.00 \\
3 & 200.72 & 194.58 & 200.72 & 211.95 & 209.80 & 1.00 \\
4 & 199.80 & 193.89 & 199.80 & 211.54 & 209.29 & 1.00 \\
5 & 198.77 & 193.11 & 198.77 & 211.07 & 208.70 & 1.00 \\
6 & 197.60 & 192.22 & 197.60 & 210.53 & 208.03 & 1.00 \\
7 & 196.26 & 191.19 & 196.26 & 209.90 & 207.26 & 1.00 \\
8 & 194.72 & 190.00 & 194.72 & 209.16 & 206.36 & 1.00 \\
9 & 192.92 & 188.59 & 192.92 & 208.28 & 205.30 & 1.00 \\
10 & 190.80 & 186.92 & 190.80 & 207.21 & 204.03 & 1.00 \\
11 & 188.26 & 184.88 & 188.26 & 205.88 & 202.48 & 1.00 \\
12 & 185.15 & 182.36 & 185.15 & 204.19 & 200.55 & 1.00 \\
13 & 181.28 & 179.15 & 181.28 & 201.96 & 198.06 & 1.00 \\
14 & 176.30 & 174.91 & 176.30 & 198.87 & 194.73 & 1.00 \\
15 & 169.63 & 169.01 & 169.63 & 194.20 & 189.99 & 1.00 \\
16 & 160.14 & 160.14 & 159.77 & 186.05 & 182.25 & 0.00 \\
17 & 144.65 & 144.65 & 138.54 & 162.58 & 159.95 & 0.00 \\
& 100.00 & 100.00 & 100.00 & & & \\
\hline
\end{tabular}

*Periods when it is optimal to enlist a broker are denoted by " 0.00 "; periods when it is optimal to continue with no broker are denoted by " 1.00 ". Parameters: $\alpha=0.95 ; \beta=1.00 ; \mathrm{u}=220.00 ; 1=150.00 ; \gamma=0.75$; $\overline{\mathrm{V}}=100.00$. 


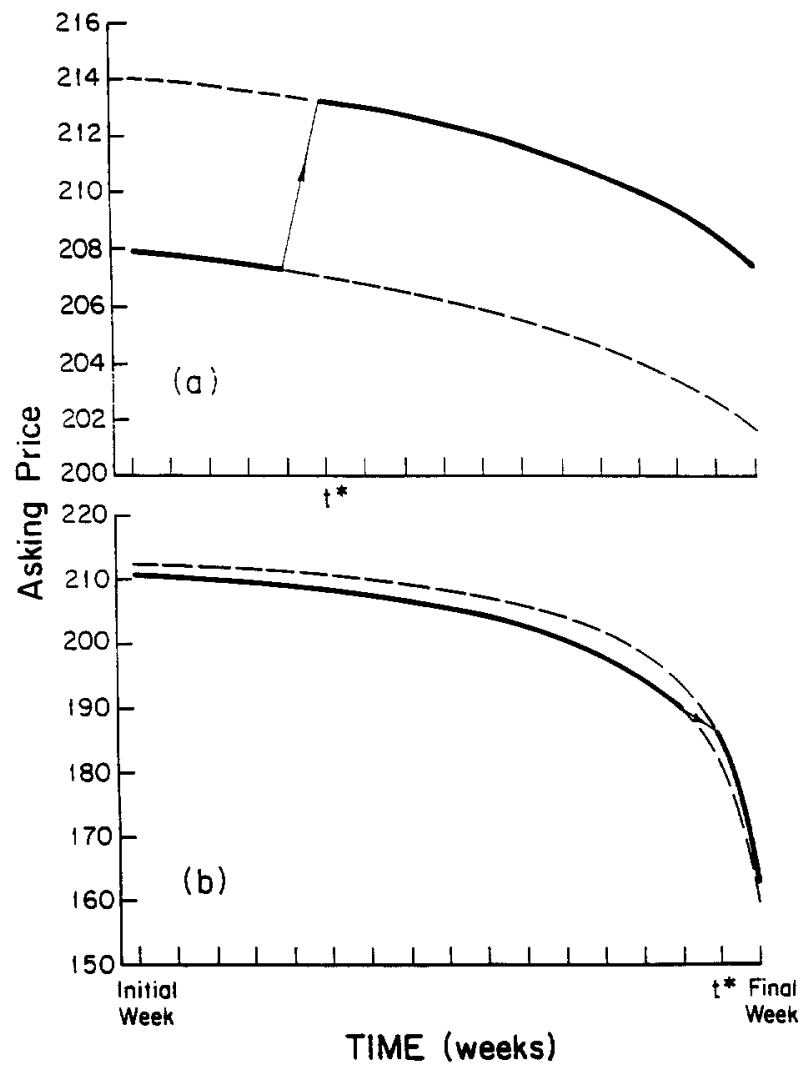

Reoltor retoined of $t^{*}$

Figure 2. Time path of asking price. (a) Upward jump in price occurs when broker is retained. (b) Upward jump fails to occur when broker is retained.

Tables 3 and 4 illustrate the sensitivity of the results to changes in the broker commission. In each of these tables, all other parameters are the same as in the benchmark case (Table 1). As Table 3 indicates, if the commission were 5\% instead, the broker would be enlisted at the outset; on the other hand, as Table 4 indicates, at an $8 \%$ commission rate, the broker would be utilized only in the final three weeks.

A similar sensitivity arises with respect to changes in $\gamma$. A reduction in $\gamma$ has no effect on the elements of $\left\{V_{t}\right\}$ but reduces the elements of $\left\{W_{t}\right\}$. The earliest date when $W_{t}=$ $V_{t}$ therefore occurs further from the terminal date $T$, and $t^{*}$ weakly decreases. Thus, if the frequency of drawing buyers in the absence of a broker were smaller, it would be optimal to terminate the by-owner phase sooner. The case where the broker is utilized from the outset arises for sufficiently low $\gamma(\gamma \leq .21$ with the other assumptions as in the benchmark case). Similarly, the case where the broker is never utilized arises for sufficiently high $\gamma(\gamma \geq$.44). 
Table 3. Base case simulation with $5 \%$ commission. Broker is retained from outset.

\begin{tabular}{ccccccc}
\hline & & & & Broker & Self & \\
Periods & $\mathrm{W}$ & $\mathrm{R}$ & $\mathrm{S}$ & $\mathrm{P}$ & $\mathrm{P}$ & Phase* \\
\hline 1 & 198.16 & 198.16 & 198.12 & 214.04 & 208.84 & 0.00 \\
2 & 197.87 & 197.87 & 197.82 & 213.87 & 208.68 & 0.00 \\
3 & 197.56 & 197.56 & 197.49 & 213.69 & 208.51 & 0.00 \\
4 & 197.22 & 197.22 & 197.13 & 213.50 & 208.32 & 0.00 \\
5 & 196.84 & 196.84 & 196.73 & 213.28 & 208.11 & 0.00 \\
6 & 196.42 & 196.42 & 196.29 & 213.03 & 207.88 & 0.00 \\
7 & 195.96 & 195.96 & 195.80 & 212.76 & 207.62 & 0.00 \\
8 & 195.45 & 195.45 & 195.25 & 212.46 & 207.34 & 0.00 \\
9 & 194.87 & 194.87 & 194.63 & 212.12 & 207.01 & 0.00 \\
10 & 194.22 & 194.22 & 193.93 & 211.73 & 206.65 & 0.00 \\
11 & 193.49 & 193.49 & 193.13 & 211.29 & 206.23 & 0.00 \\
12 & 192.65 & 192.65 & 192.22 & 210.79 & 205.75 & 0.00 \\
13 & 191.69 & 191.69 & 191.17 & 210.20 & 205.19 & 0.00 \\
14 & 190.58 & 190.58 & 189.94 & 209.52 & 204.54 & 0.00 \\
15 & 189.28 & 189.28 & 188.49 & 208.71 & 203.77 & 0.00 \\
16 & 187.73 & 187.73 & 186.74 & 207.73 & 202.85 & 0.00 \\
17 & 185.88 & 185.88 & 184.61 & 206.53 & 201.71 & 0.00 \\
& 183.60 & 183.60 & 183.60 & & & \\
\hline
\end{tabular}

*Periods when it is optimal to enlist a broker are denoted by " 0.00 "; periods when it is optimal to continue with no broker are denoted by " 1.00 ". Parameters: $\alpha=0.95 ; \beta=1.00 ; \mathrm{u}=220.00 ; 1=150.00 ; \gamma=0.25$; $\overline{\mathrm{V}}=183.60$.

Table 4. Base case simulation but with $8 \%$ commission. Broker is used sparingly.

\begin{tabular}{ccccccc}
\hline & & & \multicolumn{2}{c}{ Broker } & Self & \\
Periods & W & R & S & P & P & Phase* \\
\hline 1 & 194.80 & 192.64 & 194.80 & 214.48 & 207.11 & 1.00 \\
2 & 194.40 & 192.43 & 194.40 & 214.35 & 206.90 & 1.00 \\
3 & 193.99 & 192.20 & 193.99 & 214.22 & 206.68 & 1.00 \\
4 & 193.55 & 191.96 & 193.55 & 214.07 & 206.44 & 1.00 \\
5 & 193.08 & 191.69 & 193.08 & 213.91 & 206.20 & 1.00 \\
6 & 192.59 & 191.39 & 192.59 & 213.74 & 205.94 & 1.00 \\
7 & 192.08 & 191.07 & 192.08 & 213.54 & 205.67 & 1.00 \\
8 & 191.54 & 190.71 & 191.54 & 213.33 & 205.39 & 1.00 \\
9 & 190.97 & 190.31 & 190.97 & 213.09 & 205.09 & 1.00 \\
10 & 190.36 & 189.88 & 190.36 & 212.83 & 204.77 & 1.00 \\
11 & 189.73 & 189.39 & 189.73 & 212.53 & 204.43 & 1.00 \\
12 & 189.05 & 188.85 & 189.05 & 212.20 & 204.07 & 1.00 \\
13 & 188.33 & 188.23 & 188.33 & 211.82 & 203.69 & 1.00 \\
14 & 187.57 & 187.54 & 187.57 & 211.40 & 203.29 & 1.00 \\
15 & 186.76 & 186.76 & 186.72 & 210.91 & 202.84 & 0.00 \\
16 & 185.86 & 185.86 & 185.75 & 210.34 & 202.32 & 0.00 \\
17 & 184.82 & 184.82 & 184.61 & 209.67 & 201.71 & 0.00 \\
& 183.60 & 183.60 & 183.60 & & & \\
\hline
\end{tabular}

*Periods when it is optimal to enlist a broker are denoted by " 0.00 "; periods when it is optimal to continue with no broker are denoted by " 1.00 ". Parameters: $\alpha=0.92 ; \beta=1.00 ; \mathrm{u}=220.00 ; 1=150.00 ; \gamma=0.25$; $\overline{\mathrm{v}}=183.60$. 


\section{Future research}

The absence of much variation in broker commissions across regions of the country is a longstanding and unsolved puzzle (Owen, 1977; Wachter, 1987). The antitrust authorities, among others, have been concerned that the commission rate may reflect collusion at the national or at least the local level (Federal Trade Commission, 1984; Lesly, 1990). To investigate this hypothesis, a better understanding is needed of the derived demand for broker services. This is precisely what our model can ultimately provide. As we have seen, an increase in the commission rate causes some sellers to forego use of a real estate agent altogether and causes others to use one more sparingly. Hence, at least qualitatively, the derived demand for broker services is downward-sloping. This does not insure, however, that the substitution of by owner sales for broker sales is quantitatively significant.

Data from Canada suggest that a surprisingly large fraction of recorded house sales are by owner. According to Goldberg and Horwood (1979), during 1977 more than $25 \%$ of house sales in Montreal, Toronto, and Vancouver were by owner. Moreover, the variation in by-owner sales in the three cities is itself striking: $38.9 \%$ of sales in Montreal, $16.4 \%$ of sales in Toronto, and $23.4 \%$ of sales in Vancouver were by owner. At least for the three Canadian cities in the Goldberg-Horwood sample, the higher the city's average broker commission, the higher the percentage of sales which were by owner. These data suggest a significant substitution of owner services for broker services when commission rates are high. But, as Goldberg and Horwood point out, these data are merely suggestive. Other differences among these three Canadian cities might also account for the variation in their by-owner sales.

Once estimated, our model will permit us to quantify the effects of variations in brokers' commissions. Techniques now exist to estimate dynamic programming models such as ours from micro data - in our case, from data on asking prices, transaction prices, commission rates, elapsed time without broker and elapsed time until sale. (See, for example, GotzMcCall, 1984; Pakes, 1986; and Rust, 1987.)

When estimating this model from micro data, it is important to take explicit account of one factor from which we have abstracted - the cost to each owner in the data set of sampling another buyer without the services of a broker. Sellers who move from where their homes are located to a distant city will likely have higher costs of sampling without a broker than sellers who move around the corner. Ceteris paribus, the former group is more likely to use a broker than the latter group. But within either of these groups there will inevitably remain unobservable variations in sampling costs. After taking account of observable variations in sampling costs, the statistician should regard the unobservable component of such costs as drawn from a probability distribution. By following the dynamic behavior of a collection of individuals who have drawn the unobservable component of their sampling costs from a common distribution, the statistician can estimate its parameters.

Gotz-McCall (1984) faced similar problems in estimating their dynamic retention model for Air Force officers. They took account of heterogeneity in their sample in two ways. First, they broke the sample into nine groups based on observed differences among individuals (three distinct aeronautical rating classes and, for each, three sources of commission). Since their sample size was enormous, Gotz-McCall estimated their dynamic programming model separately for each group. ${ }^{10}$ Second, they assumed that an individual 
within any of the nine groups has an unchanging "taste for the military" known only to himself. They treated this unobservable component as drawn from a distribution common to the particular group and estimated its parameters. A similar approach could be used to estimate our search model. ${ }^{11}$ Once estimated, our model could be used to study the demand and profit consequences of variations in the commission rate.

\section{Acknowledgments}

I would like to thank Katherine Blair and Jackie Brewer for useful discussions and Patty Beeson, Dennis Capozza, Ted Sims, Joseph Swierzbinski, and Hal Varian for helpful comments on previous drafts. I would also like to thank Pat O'Connell-Young and Eula Poole for their timely assistance on the final draft.

\section{Notes}

1. Since, for any solution to our model, there is an exogenous rental rate which rationalizes our solution as a typical cycle in a model with an endless succession of cycles, nothing of importance is lost by this simplification.

2. A second source of nonstationarity from which we abstract here is that the seller is initially uncertain about relevant market parameters and learns about them as he samples.

3. Indeed, as Lesly (1990) writes, rumors abound that the FTC is once again investigating the apparent invariance of commission rates and is about to bring an antitrust case against the National Association of Realtors.

4. To explain why a given individual might begin selling "by owner" but might later switch to a broker, we have simplified; we have assumed that the cost of sampling buyers is zero. In reality, of course, such costs are nonzero and sometimes govern whether or not an owner uses a broker. Moreover, variations in these costs across different individuals help explain why some sellers retain a broker while others do not. Hence, as discussed in the concluding section, the cost to the owner of sampling buyers without a broker must be taken into consideration when estimating the model, using observed behavior of heterogeneous individuals.

5. We have drawn $R(V)$ as strictly convex, since, as can be verified from (4), it has this curvature. However, the existence of a unique fixed point in $(0, \alpha u)$ does not depend on this characteristic; hence the verification of the curvature has been left to the reader.

6. By $R^{k}(\bar{V})$ is meant the application of the function $R$ to the real number $\bar{V}$ to produce the real number $R(\bar{V})$, the application of the function $R$ to this result, and so forth for a total of $k$ applications of the function. Thus, $R^{2}(\bar{V})=R(R(\bar{V}))$.

7. For $S(c)$ must be at least as large as the right-hand side of $(10)$ evaluated at the suboptimal $\hat{P}^{*}(b)$ and that strictly exceeds $S(b)$.

8. In terms of Figure 1, an upward jump would be inevitable if $\bar{V}$ lies to the right of the intersection of the convex curve $(R(V))$ and a ray through the origin with slope $1 / \alpha$. Varying $\gamma$ to insure the optimality a two-phase program will shift neither the ray nor $R(V)$.

9. For simplicity, we have assumed in the simulations that one buyer always arrives during the period if the broker is engaged $\left(\gamma_{R}=1\right)$. Hence, $\gamma=\gamma_{S}$ and $f(y)=g(y)$.

10. Since our sample will be much smaller, a more efficient approach would be needed to account for observed heterogeneity.

11. In our application, one might want to assume instead that the unobservable component of the sampling cost, once drawn by the seller, evolves from its initial value according to a simple Markov process as the season progresses.

\section{References}

Federal Trade Commission. Residential Real Estate Brokerage Industry, Washington, D.C.: (2 volumes), 1984. 
Goldberg, M.A. and Horwood, P. "Housing Transaction Costs: Evidence from Three Canadian Cities," report submitted to Canadian Department of Consumer and Corporate Affairs, Ottawa, Ontario, 1979.

Gotz, G. and McCall, J. "A Dynamic Retention Model for Air Force Officers" RAND Report R-3028-AF, 1984.

Lesly, E. "How Your Realtor Rips You Off," The Wall Street Journal, November 30, 1990, p. A14.

Owen, Bruce M. "Kickbacks, Specialization, Price Fixing, and Efficiency in Residential Real Estate Markets," Stanford Law Review 29, May 1977, 931-967.

Pakes, A. "Patents as Options: Some Estimates of the Value of Holding European Patent Stocks," Econometrica, 54, 1986, 755-84.

Rust, J. "Optimal Replacement of GMC Bus Engines: An Empirical Model of Harold Zurcher," Econometrica, 55, 1987, 999-1034.

Wachter, Susan M. "Residential Real Estate Brokerage: Rate Uniformity and Moral Hazard," Research in Law and Economics, 10, 1987, 189-210. 